\title{
Freidenberg, Flavia y Muñoz-Pogossian, Betilde (eds.), Reformas a las organizaciones de partidos en América Latina (1978-2015), Lima, Pontificia Universidad Católica del Perú, Escuela de Gobierno y Políticas Públicas- UNAM, Instituto de Investigaciones Jurídicas- OEA-Sociedad Argentina de Análisis Político,
} 2016, $521 \mathrm{pp}$.

A cuatro décadas del inicio de las transiciones a la democracia en América Latina, los países de la región enfrentan los retos de mantener gobiernos estables e inclusivos en contextos permeados por altos niveles de desigualdad, inseguridad y corrupción, entre otros problemas. Ese escenario tiene como actores protagónicos a los partidos políticos, los cuales, aun con las reacciones negativas que generan, son indispensables en el juego democrático. Por tal motivo, y a pesar de las voces que apelan por su debilitamiento en favor de liderazgos personalistas y mecanismos de democracia directa, resulta imprescindible saber cómo el cambio en el diseño institucional puede favorecer o debilitar estructuras y prácticas partidarias en pro de los derechos humanos, de una mayor participación ciudadana y de mejores sistemas de rendición de cuentas.

La presente obra constituye una sólida y amplia aportación para comprender los problemas que enfrentan (y producen) los partidos políticos en las democracias latinoamericanas, y la manera en que las reformas políticas han incidido en sus organizaciones, muchas veces con objetivos claros y resultados positivos, pero otras tantas con metas cortoplacistas y desestructuradas que han contribuido a debilitar la representación política y la coordinación gubernamental. En ese marco, es indispensable ubicar la discusión desde un punto de partida que señalan las editoras del libro: las reformas político-electorales tienen un efecto en las organizaciones partidarias, pero ¿de qué profundidad son las repercusiones?, ¿qué ámbitos tocan?, ¿cómo se relacionan con otras variables para produ- 
cir diferentes resultados?, ¿cómo son los procesos para modificar las normas?, ¿cuáles son los aprendizajes de las últimas décadas en la materia? y ¿qué tanta debe ser la intervención estatal en los partidos?

A través de 19 capítulos, 23 especialistas, de la academia y del ámbito profesional, apuntan las principales tendencias sobre las reformas electorales en la región, y su lugar en la agenda de investigación y en la discusión pública. La obra destaca por el diálogo interdisciplinario entre cada uno de sus capítulos: tiene importantes contribuciones desde la perspectiva jurídica, al hacer precisas descripciones normativas y proponer nuevas definiciones y argumentaciones en materia electoral, pero también hay una constante retroalimentación desde la ciencia política comparada, en un plano teórico y contextual. El resultado de cada uno de los textos permite elaborar recomendaciones para políticas públicas con un sustento empírico, y plantear nuevas preguntas de investigación sobre temas de los cuales aún hay vacíos. El libro está dividido en cuatro partes, todas referidas a las organizaciones de partidos: 1) Reformas a los procesos de selección de candidaturas; 2) Reformas a los mecanismos de acción afirmativa, y promoción de los liderazgos de las mujeres; 3 ) Reformas a la financiación de los partidos; y 4) Reformas a los sistemas electorales, a partir de sus efectos sobre el funcionamiento de estas organizaciones.

La sección sobre los cambios en los procesos de nominación al interior de los partidos cobra relevancia en cuanto a que ha sido una reforma que ha buscado expandir los derechos políticos de los militantes y promover una mayor democracia, pero que tiene importantes repercusiones para las organizaciones. Esa discusión, que parte de dicha tensión intrínseca, es expuesta en cinco trabajos. Flavia Freidenberg, editora del libro e investigadora del Instituto de Investigaciones Jurídicas (IIJ) de la UNAM, presenta un minucioso mapa de las normas sobre las elecciones al interior de los partidos políticos y sus cambios. Esa revisión, como señala la autora, evidencia una amplia heterogeneidad y permite plantear que, si bien los comicios internos son deseables, por sí solos no garantizan una mayor democracia interna. Lo que suele ocurrir es que dichas elecciones potencian conflictos internos y una mala percepción en el exterior. Para que éstas tengan un efecto positivo, apunta la investigadora, deben acompañarse de condiciones que apelan a una mayor cultura democrática entre miembros y dirigentes de los partidos.

Desde el ámbito de la justicia electoral, María del Carmen Alanís Figueroa, magistrada del Tribunal Electoral del Poder Judicial de la Federación (TEPJF) de México, analiza el rol que los mecanismos y los órganos jurisdiccionales juegan para garantizar procesos democráticos al interior 
de los partidos políticos. La autora presenta las dos tradiciones jurídicas al respecto y sus problemas, una liberal y otra con mayor intervención estatal en dichas organizaciones, y argumenta que la inclusión de un elemento democrático en la regulación sobre los procesos, al interior de los partidos, ha implicado la expansión del ámbito jurisdiccional, lo cual conlleva una acción positiva para evitar la discriminación y la subrepresentación de segmentos de la ciudadanía en el proceso político.

César Astudillo Reyes, investigador del IIJ de la UNAM, presenta una revisión de las normas sobre nominaciones independientes y por partidos desde la perspectiva de los derechos de participación política. El autor señala que el derecho al voto se ha convertido en un instrumento de legitimación democrática, en ese contexto es esencial que esta prerrogativa sea revisada y profundizada en sus distintas dimensiones, tanto en la activa como en la pasiva; sin embargo, si bien su expansión es deseable al eliminar la mayor parte de los obstáculos para su ejercicio, específicamente en el caso de las candidaturas independientes, no se puede perder de vista las implicaciones y las limitaciones razonables que deben tener por su repercusión para el sistema político en su conjunto.

Los dos textos que complementan la sección presentan casos nacionales analizados a profundidad. Salvador Romero Ballivián, director del Centro de Asesoría y Promoción Electoral del Instituto Interamericano de Derechos Humanos, hace una detallada descripción de las elecciones primarias en Honduras, su aporte reside en el seguimiento de una implementación de elecciones internas que se ha mantenido por más de 25 años. En dicho caso, resaltan sus características: voluntarias, simultáneas, a padrón abierto, para todos los cargos y con responsabilidad administrativa y jurisdiccional del Tribunal Superior Electoral. A partir de dichos elementos, el estudio del caso hondureño permite conocer una experiencia exitosa, y los temas pendientes por revisar. Por su parte, Alejandro Tullio, titular de la Dirección Nacional Electoral de Argentina, observa la reforma al proceso de selección de candidaturas nacionales en su país. El autor hace una revisión crítica de la norma que en 2009 introdujo un sistema de elecciones primarias, abiertas, simultáneas y obligatorias con el cual hubo efectos por la proliferación de partidos políticos y la separación de fechas para la realización de los comicios. Desde su perspectiva, el sistema no contribuyó a fortalecer un régimen político que estaba en crisis. El texto permite identificar las externalidades negativas que pueden tener modificaciones de tal calado, y las motivaciones de ellas.

En la segunda parte, se presentan cinco trabajos sobre las modificaciones a los mecanismos de acción afirmativa y promoción de los liderazgos 
de las mujeres en los partidos. Mariana Caminotti, académica de la Universidad Nacional de San Martín, presenta el panorama de la adopción de las cuotas de género y paridad en América Latina, y evalúa su efectividad. Al respecto, la autora elabora un sólido argumento: las normas fortalecieron la protección de los derechos políticos de las mujeres, sin embargo, la igualdad sustantiva enfrenta barreras estructurales y la subsistencia de reglas informales. De esa forma, resalta la necesidad de establecer ciertas reglas, pero acompañadas con otras medidas.

Betilde Muñoz-Pogossian, editora del libro y directora del Departamento de Inclusión Social de la OEA, analiza los modelos de reclutamiento de los partidos políticos en América Latina, y la representación de las mujeres en dichas organizaciones. Parte de que los partidos son el punto de entrada para los puestos de poder, describe la situación de las mujeres de la región en ellos, caracterizada por el estancamiento, y propone un modelo conceptual para estudiar el fenómeno. Al final, señala la pertinencia, entre otras medidas, de trabajar en el acceso de las mujeres a medios de comunicación para hacer campaña y la disponibilidad de financiamiento para fortalecer su participación.

La importancia de la estructura organizativa de los partidos para la inclusión de las mujeres es estudiada por Margarita Batlle, profesora de la Universidad Externado de Colombia, quien a través del análisis documental y de entrevistas cualitativas presenta el caso de los partidos colombianos. La autora señala que, a pesar de la inclusión de la cuota de género en 2011, las organizaciones políticas no se han adaptado para potenciar el fortalecimiento de la participación de las mujeres. Su texto evidencia que la implementación de las cuotas no necesariamente se refleja en acciones de los partidos, en los que, señala la autora, aún no hay medidas claras para atender el tema.

Para completar la sección, dos capítulos analizan experiencias nacionales de la evolución normativa en la materia y el papel que juegan las autoridades electorales para su efectiva implementación. Alberto R. Dalla Via, vicepresidente de la Cámara Nacional Electoral de Argentina, hace un recuento de la participación política de las mujeres en su país, centrándose principalmente en la evolución jurídica; cabe recordar que Argentina fue en 1991 la primera nación de América Latina que incluyó las cuotas de género en sus normas. El autor resalta el papel de los organismos electorales, especialmente de los jueces, para aplicar la normatividad, y argumenta que la evaluación del éxito de las medidas debe ir más allá de la mera revisión cuantitativa. En un sentido parecido, Luis Antonio Sobrado González, magistrado del Tribunal Superior de Elecciones 
de Costa Rica, presenta el caso de su país en la implementación de la cuota de género, incluida en la normatividad desde 1996. En su estudio, también resulta evidente la importancia de que los jueces electorales estén comprometidos con el objetivo de las leyes, ya que la jurisprudencia en la materia sirvió para lograr que se cumpliera lo que dictaba una norma susceptible de no ser puesta en práctica de forma cabal. De esa manera, la figura del activismo judicial para la consecución de los objetivos constituye un elemento primordial para lograr avances en esta área.

La tercera sección analiza las reformas al financiamiento de los partidos en la región. Kevin Casas-Zamora y Daniel Zovatto, de Interamerican Dialogue e IDEA Internacional, respectivamente, describen la situación en América Latina, y recuerdan que la relación dinero-política, más que una patología, es parte de la vida democrática. El texto sirve como una actualización del trabajo que ambos autores han realizado previamente, con un énfasis en los riesgos asociados al financiamiento, como la inmersión del narcotráfico en las elecciones. La experiencia acumulada les permite formular un conjunto de propuestas al momento de plantear una reforma, entre ellas una guía para su inicio y un esquema de evaluación. Su lectura resultará esencial para quienes estén interesados en el proceso de modificación y revisión de las leyes en la materia.

Claudio Fuentes y Mario Herrera, de la Universidad Diego Portales de Chile, estudian la relación entre el financiamiento político y la calidad de la democracia. Para ello brindan una revisión de la literatura especializada, detallando un conjunto de aristas que van desde la comparación de los marcos legales hasta las implicaciones causales. Dentro de ellas, destacan que el estudio se ha centrado más en las normas y menos en la valoración de los efectos de las medidas, y del impacto real del dinero; en este último rubro, señalan un amplio campo por indagar. De especial atención es su propuesta de índice, para evaluar y comparar las diferentes estructuras de financiamiento en la región.

Ciro Murayama, consejero del Instituto Nacional Electoral de México, presenta el caso del país en materia de financiamiento político y acceso a medios de comunicación. El también académico analiza sus principales características legales, enfocadas en aspectos clave y que buscan equi$\mathrm{dad}$, independencia y transparencia entre los actores políticos. Asimismo, ahonda en la modificación de 2014, que produjo un aumento en los recursos públicos para los partidos en el nivel subnacional. Con el análisis se puede comprender la importancia de tales reformas para impulsar cambios democratizadores. 
La cuarta parte examina la relación entre las reformas a los sistemas electorales, y sus efectos en el funcionamiento de las organizaciones de partidos. Para iniciar, Daniel Buquet, profesor del Instituto de Ciencia Política de la Universidad de la República de Uruguay, plantea la discusión teórica sobre las organizaciones partidarias, y recuerda que pocas veces ésta se ha vinculado expresamente al diseño institucional en el que compiten. Sin embargo, el autor argumenta que hay experiencias y planteamientos analíticos, para sostener que existe ese vínculo, específicamente a través de la generación de incentivos. Para probarlo, identifica el grado de centralización de los partidos, especialmente en sistemas presidenciales, y si éstos se ven afectados por el tipo de normas electorales. Encuentra, parcialmente, evidencia para sostener esa relación. El análisis sirve para mostrar la dificultad para aislar esos efectos $y$, por ende, predecir los resultados de ciertas normas.

Otro ángulo es abordado por María del Socorro Sousa Braga, investigadora de la Universidad Federal de San Carlos de Brasil, quien presenta los efectos institucionales, pero en el desarrollo organizativo de los partidos en América Latina. La autora indica que las reformas de las últimas décadas han hecho más inclusivos a los sistemas, pero también han acrecentado la relación entre electores y representantes, especialmente con un vínculo personalizado. Para adentrarse en dicho fenómeno, sigue a los países de la región que pasaron a tener gobiernos de izquierda: Bolivia, Venezuela, Chile y Brasil. Los hallazgos del trabajo abonan a áreas de estudio sobre las que, como ella misma presenta, ha habido un mínimo avance en su conocimiento.

Fernando Tuesta Soldevilla, académico de la Pontificia Universidad Católica de Perú, analiza el papel que las reglas electorales tuvieron en la proliferación de los partidos en el país andino. Asimismo, expone cómo la fragmentación del sistema de partidos peruano ha conllevado una serie de problemas, de tal suerte que en ese contexto las modificaciones a las leyes han producido un mayor debilitamiento. La situación ha sido resultado de reformas sin un plan, un sistema de partidos desvinculado de la bonanza económica, problemas de organización de los partidos, un alto personalismo en la política, y un sistema susceptible al dinero ilícito. Esas medidas han implicado una representación con problemas para llegar a acuerdos al momento de negociar.

José Ricardo Puyana Valdivieso (profesor de la Universidad del Externado de Colombia), Mario Alberto Ruiz y Daniela Lucía Vargas (del Programa de las Naciones Unidas para el Desarrollo), presentan el panorama de las reformas electorales en Colombia. En este caso, las modificaciones 
han sido en sentido contrario a Perú, al generar un sistema más consolidado, nacionalizado y con menores niveles de volatilidad. Los autores hacen un recorrido a partir del cambio de 2003. Los resultados, señalan, fueron inmediatos, al fortalecer a las organizaciones, pero aún persisten retrasos en temas internos y de equidad en la contienda electoral.

En esta última sección, se incluyen dos trabajos que analizan temas particulares que inciden directa e indirectamente en las organizaciones políticas. Alejandro Luna Ramos, exmagistrado del TEPJF, presenta las reformas a la transparencia de los partidos en México, para lo cual contextualiza el acceso a la información y al gobierno abierto como luchas que formaron parte de la consolidación democrática. Sin embargo, indica que el cumplimento de esas garantías se alcanzó tres décadas después, en una tarea acompañada por un andamiaje institucional que ha requerido de autoridades que aplican las normas para salvaguardar tales derechos.

El otro cambio es la inclusión del voto migrante. Dicho análisis es de Yuri Gabriel Beltrán Miranda, consejero del Instituto Electoral del Distrito Federal, quien en un primer momento presenta el debate doctrinario que implica dicho derecho, y las complejidades técnicas para su puesta en práctica, especialmente cuando se introduce esa prerrogativa. El especialista describe la adopción de la medida en las normas de los países de la región, y analiza cómo reaccionan lo partidos políticos ante ellas, resaltando los esfuerzos para buscar a esos votantes. Concluye que la implementación produce incentivos para el cambio en los partidos, pero la respuesta no es homogénea. Finalmente, propone evaluar el éxito de la aplicación, no sólo por el número de votos recibidos y su costo, sino también por la inclusión de los migrantes en las vidas de estas organizaciones.

Como se señala al inicio del libro, las reformas son prácticas comunes en los regímenes actuales, y tienen efectos en su funcionamiento. La obra cumple al ofrecer una visión de su impacto en los partidos políticos, de manera general; y en temas puntuales, para mejorar la calidad de las democracias. Sus contribuciones son de utilidad para la investigación académica, para los tomadores de decisiones y para los interesados en la materia.

\section{Raymundo Alva Huitrón*}

\footnotetext{
* Estudiante de la Maestría en Gobierno y Asuntos Públicos del Programa de Posgrado en Ciencias Políticas y Sociales de la UNAM.
} 